\title{
Quark number susceptibility of high temperature and finite density QCD.
}

\author{
A. Hietanen* \\ Theoretical Physics Division, Department of Physical Sciences P.O. Box 64 FI-00014 University \\ of Helsinki, Finland and \\ Helsinki Institute of Physics, P.O. Box 64, FI-00014 University of Helsinki, Finland \\ E-mail: ari.hietanen@helsinki.fi

\section{K. Rummukainen} \\ Department of Physics, University of Oulu P.O. Box 3000, FI-90014 Oulu \\ E-mail: kari.rummukainen@oulu.fi
}

\begin{abstract}
We utilize lattice simulations of the dimensionally reduced effective field theory (EQCD) to determine the quark number susceptibility of QCD at high temperature $\left(T>2 T_{C}\right)$. We also use analytic continuation to obtain results at finite density. The results extrapolate well from known perturbative expansion (accurate in extremely high temperatures) to $4 \mathrm{~d}$ lower temperature lattice data.
\end{abstract}

The XXV International Symposium on Lattice Field Theory

July 30-4 August 2007

Regensburg, Germany

\footnotetext{
${ }^{*}$ Speaker.
} 


\section{Introduction}

The quark number (baryon number) susceptibility is an observable which is one of the signature of the quark-gluon plasma in heavy ion collision experiments [1]. Thus, it is of interest to accurately calculate the quark number susceptibility theoretically. In weak-coupling perturbation theory, the susceptibility of the quark-gluon plasma has been calculated up to order $g^{6} \ln 1 / g$ [2]. Because of the asymptotic freedom, at high enough temperatures the perturbation theory is a valid approach. However, the convergence of the perturbative series is bad at physically accessible temperatures, and the applicability of the results is not obvious, see Fig. 1. The order-by-order behaviour of the susceptibility is not systematic, and the low-temperature behaviour changes qualitatively at orders $g^{3}$ and $g^{5}$. Further, if we allow variations in the unknown $O\left(g^{6}\right)$-coefficient in the expansion (Fig. 1 right), we obtain quite large variation in the final result at temperatures under $10 T_{c}$.

The quark number susceptibility has also been studied with lattice simulations [3, 4]. While the standard lattice methods (with dynamical fermions) are the best method to study QCD in the immediate vicinity of the phase transition, at higher temperatures it is more economical to simulate dimensionally reduced effective theory, electrostatic QCD (EQCD) [5]. This method has already been used to calculate the pressure in quark-gluon plasma [6]. Here we present the updated results of simulations of quark number susceptibility for $N_{f}=2$ [7] for zero chemical potential. We also present preliminary results from simulations extended to finite chemical potential. These results are obtained by doing simulations with imaginary values of the chemical potential and then analytically continuing to the real values. This is achieved by fitting a polynomial of $\mu^{2}$ to the data.
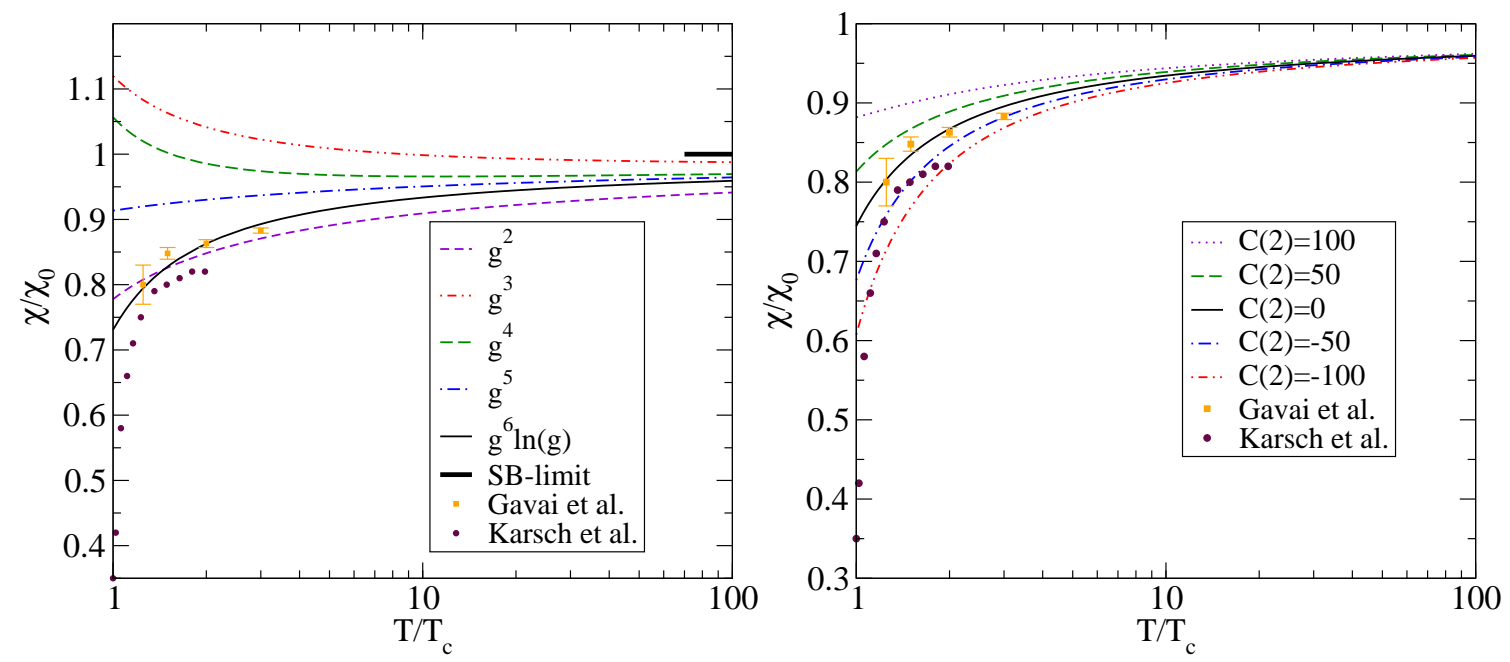

Figure 1: Left: The perturbative expansion of quark number susceptibility order by order for $N_{f}=2$. The coefficient at order $g^{6}$ has been fixed here to match the lattice measurements. Right: The effect of changing the value of the unknown $O\left(g^{6}\right)$ coefficient, parametrised as $C\left(N_{f}\right)\left(\frac{g^{2}}{4 \pi^{2}}\right)^{3}$. The perturbative results are from [2] and the lattice results from [3, 4]. 


\section{Susceptibility in electrostatic QCD}

EQCD is defined by the action

$$
S_{\mathrm{E}}=\int \mathrm{d}^{3} x\left\{\frac{1}{2} \operatorname{Tr}\left[F_{i j}^{2}\right]+\operatorname{Tr}\left[D_{i}, A_{0}\right]^{2}+m_{3}^{2} \operatorname{Tr}\left[A_{0}^{2}\right]+i \gamma_{3} \operatorname{Tr}\left[A_{0}^{3}\right]+\lambda_{3}\left(\operatorname{Tr}\left[A_{0}^{2}\right]\right)^{2}\right\},
$$

where $F_{i j}=\partial_{i} A_{j}-\partial_{j} A_{i}+i g_{3}\left[A_{i}, A_{j}\right]$ and $D_{i}=\partial_{i}+i g_{3} A_{i} . F_{i j}, A_{i}$ and $A_{0}$ are traceless $3 \times 3$ Hermitean matrices $\left(A_{0}=A_{0}^{a} T_{a}\right.$, etc). The coupling and the mass parameters $g_{3}, m_{3}, \gamma_{3}$ and $\lambda_{3}$ are determined by the physical $4 \mathrm{~d}$ temperature, renormalization scale $\Lambda_{\overline{\mathrm{MS}}}$, chemical potential $\mu$ and the number of massless fermions. It is convenient to use the dimensionless ratios

$$
y=\frac{m_{3}^{2}}{g_{3}^{4}}, \quad x=\frac{\lambda_{3}}{g_{3}^{2}}, \quad z=\frac{\gamma_{3}}{g_{3}^{3}}
$$

which determine the physical properties of EQCD. The $\mu$-dependence of the parameters is, at 1loop level,

$$
y=y_{\mu=0}\left(1+\sum_{f} \bar{\mu}_{f}^{2} \frac{3}{2 N_{\mathrm{c}}+N_{\mathrm{f}}}\right), \quad z=\sum_{f} \frac{\bar{\mu}_{f}}{3 \pi}, \quad x=x_{\mu=0},
$$

where $\bar{\mu}=\mu /(\pi T)$ and the $\mu=0$ expressions can be found in ref. [5]. The two loop corrections have been calculated in ref. [8], but the effects remain in practice negligible.

The quantity we are interested in is the quark number susceptibility, which we define as a derivative over one flavor $u$ only:

$$
\chi_{3} \equiv \frac{1}{V} \frac{\partial^{2}}{\partial \mu_{u}^{2}} \ln \mathscr{Z}=\frac{1}{V} \frac{\partial^{2}}{\partial \mu_{u}^{2}} \ln \int \mathscr{D} A_{k} A_{0} \exp \left(-S_{\mathrm{E}}\right)
$$

Substituting $S_{\mathrm{E}}$ from (2.1) we arrive at the result

$$
\begin{aligned}
\chi_{3}= & -\frac{6}{2 N_{c}+N_{f}} y_{\mu=0}\left\langle\operatorname{Tr} A_{0}^{2}\right\rangle \\
& +\frac{1}{V 9 \pi^{2}} \int d^{3} r_{1} d^{3} r_{2}\left(\left\langle\operatorname{Tr} A_{0}^{3}\left(r_{1}\right) \operatorname{Tr} A_{0}^{3}\left(r_{2}\right)\right\rangle-\left\langle\operatorname{Tr} A_{0}^{3}\right\rangle^{2}\right) \\
& +\frac{36}{V\left(2 N_{c}+N_{f}\right)^{2}} \bar{\mu}_{u}^{2} y_{\mu=0}^{2} \int d^{3} r_{1} d^{3} r_{2}\left(\left\langle\operatorname{Tr} A_{0}^{2}\left(r_{1}\right) \operatorname{Tr} A_{0}^{2}\left(r_{2}\right)\right\rangle-\left\langle\operatorname{Tr} A_{0}^{2}\right\rangle^{2}\right) .
\end{aligned}
$$

Thus, the quark number susceptibility is obtained by measuring the condensates $\left\langle\operatorname{Tr} A_{0}^{2}\right\rangle,\left\langle\left(\operatorname{Tr} A_{0}^{2}\right)^{2}\right\rangle$ and $\left\langle\left(\operatorname{Tr} A_{0}^{3}\right)^{2}\right\rangle$ on the lattice. Due to the superrenormalizable nature of the theory, measurements can be rigorously converted to $\overline{\mathrm{MS}}$ scheme in the lattice continuum limit, and because $\overline{\mathrm{MS}}$ was used in the perturbative matching to $4 \mathrm{~d} \mathrm{QCD}$, this also allows us to compare to $4 \mathrm{~d}$ results.

The lattice counterterms needed for the continuum limit of $\left\langle\operatorname{Tr} A_{0}^{2}\right\rangle$ are given in [5], and of $V\left\langle\operatorname{Tr} A_{0}^{3}\left(r_{1}\right) \operatorname{Tr} A_{0}^{3}\left(r_{2}\right)\right\rangle$ in [7]. The contribution including $\left\langle\operatorname{Tr} A_{0}^{2}\left(r_{1}\right) \operatorname{Tr} A_{0}^{2}\left(r_{2}\right)\right\rangle$ is not UV divergent and thus does not require counterterms.

Finally, the relation between $\chi_{3}$ and the true $4 \mathrm{~d}$ susceptibility is

$$
\chi=\frac{g_{3}^{6}}{T^{3}} \chi_{3}+\frac{\partial^{2}}{\partial \mu_{u}^{2}} \Delta p
$$

where $\Delta p=p_{\mathrm{QCD}}-p_{3 \mathrm{~d}}$ is the perturbative $3 \mathrm{~d} \rightarrow 4 \mathrm{~d}$ matching coefficient for pressure, and can be found in [9]. 

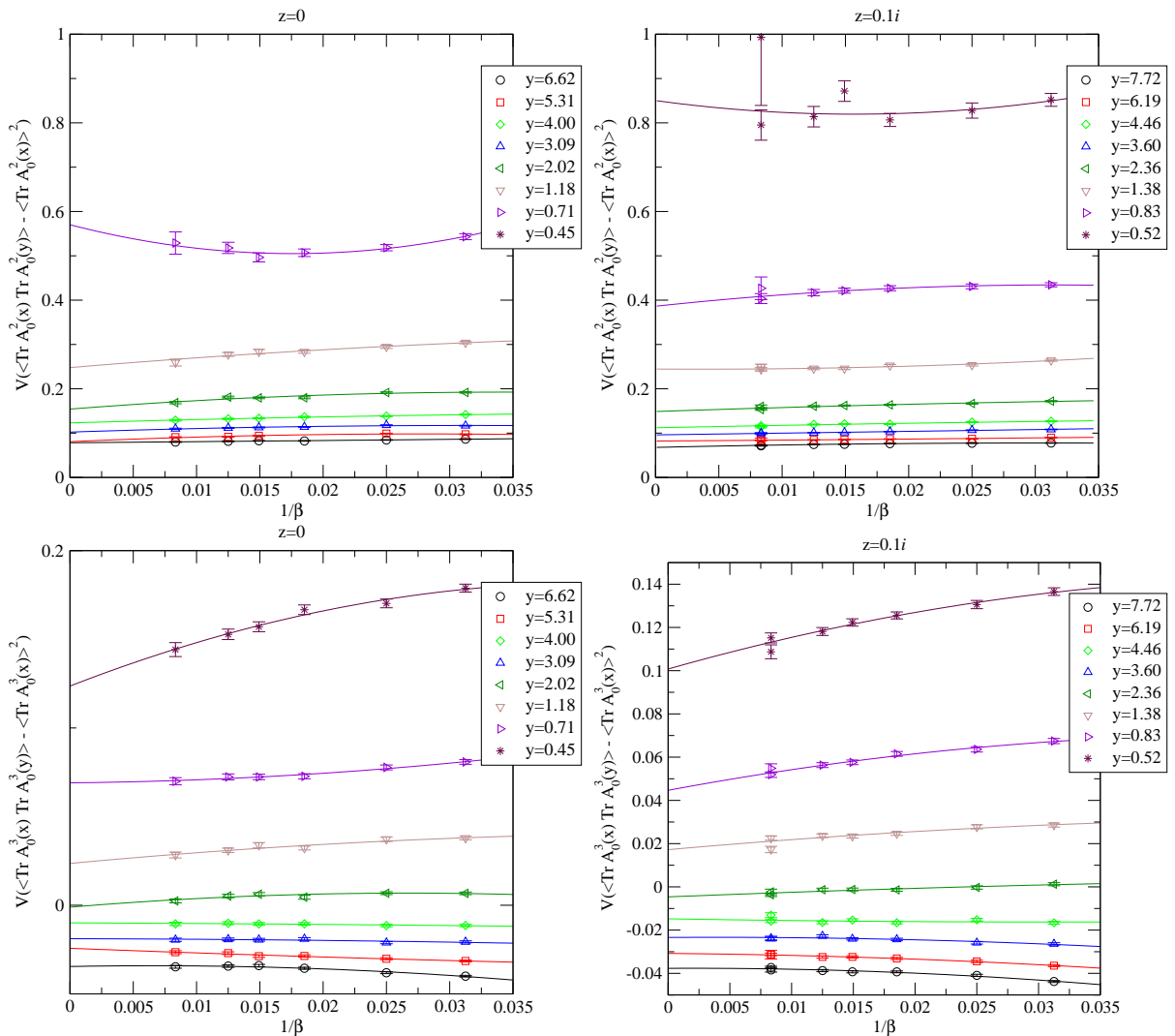

Figure 2: Continuum extrapolations of $V\left(\left\langle\left(\operatorname{Tr} A_{0}^{2}\right)^{2}\right\rangle-\left\langle\operatorname{Tr} A_{0}^{2}\right\rangle^{2}\right)$ and $V\left(\left\langle\left(\operatorname{Tr} A_{0}^{3}\right)^{2}\right\rangle-\left\langle\operatorname{Tr} A_{0}^{3}\right\rangle^{2}\right)$.

\section{Lattice measurements}

Lattice simulations are carried out for $N_{\mathrm{f}}=2$. We use 6 different values of chemical potential $\mu$, and for each value of $\mu$ we use eight different values of temperature $T$. For each of these $(\mu, T)$ pairs we use five different values of the lattice spacing $a$, in order to obtain a reliable continuum limit. To check the finite volume effects we did simulations with different volumes at the smallest lattice spacing; for a detailed analysis in a related theory see [10].

Precise continuum limits are necessary for accurate determination of $\chi_{3}$. For the condensate $\left\langle\operatorname{Tr} A_{0}^{2}\right\rangle$ we use a fit ansatz of form

$$
c_{1}+\frac{c_{2}}{\beta}+\frac{c_{2}^{\prime}}{\beta} \log (\beta)+\frac{c_{3}}{\beta^{2}},
$$

where $\beta=6 /\left(g_{3}^{2} a\right)$. The existence of the logarithmic term increases the final errors significantly. However, the coefficients of the above ansatz are perturbatively calculable, and there is an ongoing program to determine the coefficient of the log-term using stochastic perturbation theory [11]. The knowledge of this would reduce the errors by order of magnitude.

The contributions $V\left(\left\langle\left(\operatorname{Tr} A_{0}^{2}\right)^{2}\right\rangle-\left\langle\operatorname{Tr} A_{0}^{2}\right\rangle^{2}\right)$ and $V\left(\left\langle\left(\operatorname{Tr} A_{0}^{3}\right)^{2}\right\rangle-\left\langle\operatorname{Tr} A_{0}^{3}\right\rangle^{2}\right)$ are fitted with second order polynomial ansatz

$$
c_{1}+\frac{c_{2}}{\beta}+\frac{c_{3}}{\beta^{2}} .
$$



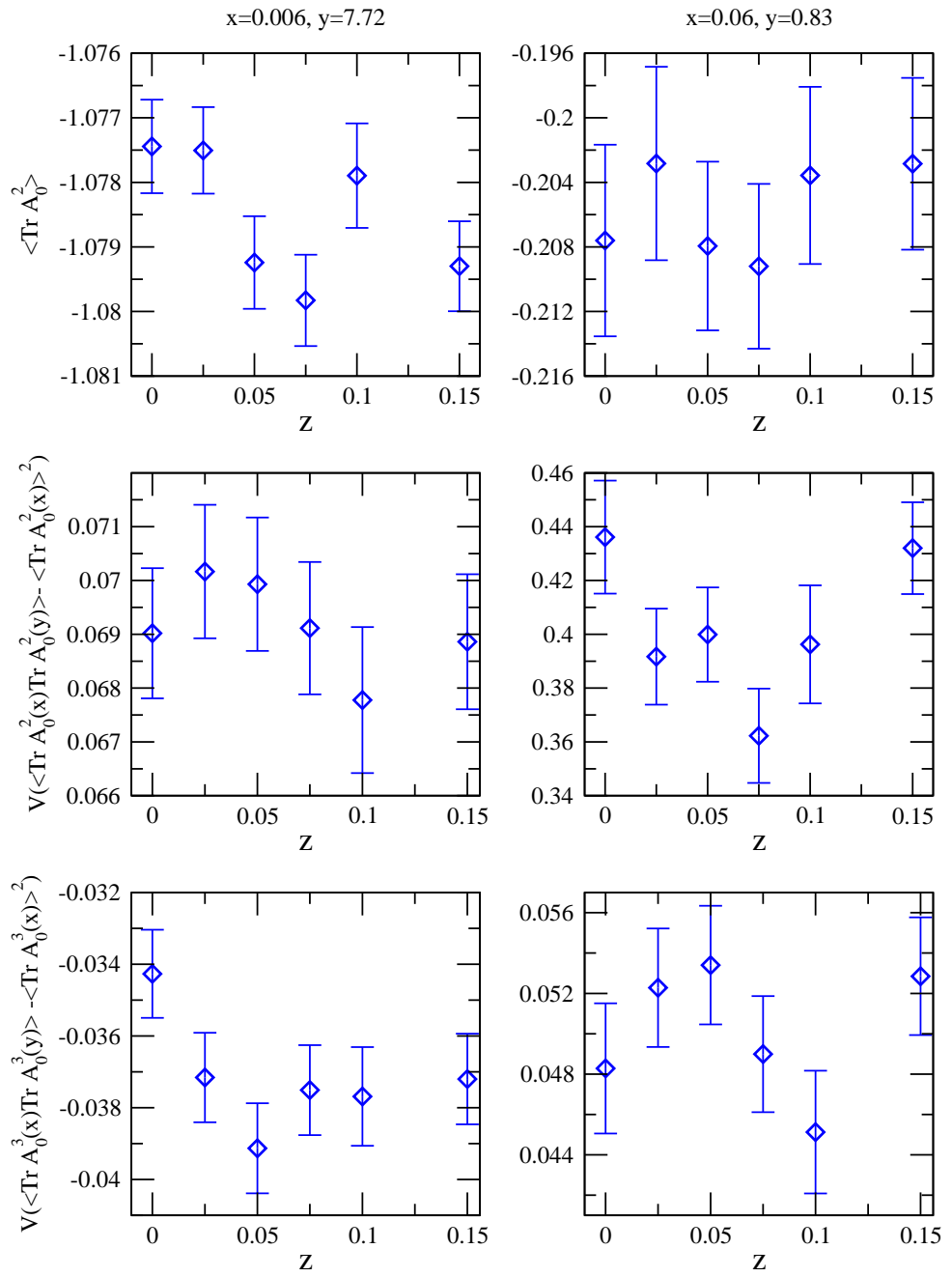

Figure 3: Simulations done with two different $(x, y)$-pairs while varying the imaginary chemical potential in the range $0 \leq-i z \leq 0.15$. Within our accuracy varying $i z$ does change the results, making analytic continuation straightforward.

This fits the data well, see Fig. 2.

We study the chemical potential dependence by using imaginary $\mu$ and performing analytical continuation. However, this turns out to be rather trivial: for fixed $x, y$ the dependence of the results on $i z \propto i \mu$ is very small and not visible within our statistical errors, see Fig. 3. (However, see the note above about the statistical errors in $\left\langle A_{0}^{2}\right\rangle$.) Thus, the $\mu$-dependence of the results is, in practice, completely due to the $\mu$-dependence of $y$.

The final continuum extrapolated results agree well with the perturbative susceptibility. It is of the form

$$
\chi_{\text {pert }}=a_{1} y^{3 / 2}+a_{2} y+a_{3} y^{1 / 2}+a_{4}
$$

Hence the difference of lattice and perturbation theory should behave as $y^{-1 / 2}$, which is the case, as can be seen in Fig. 3 . 

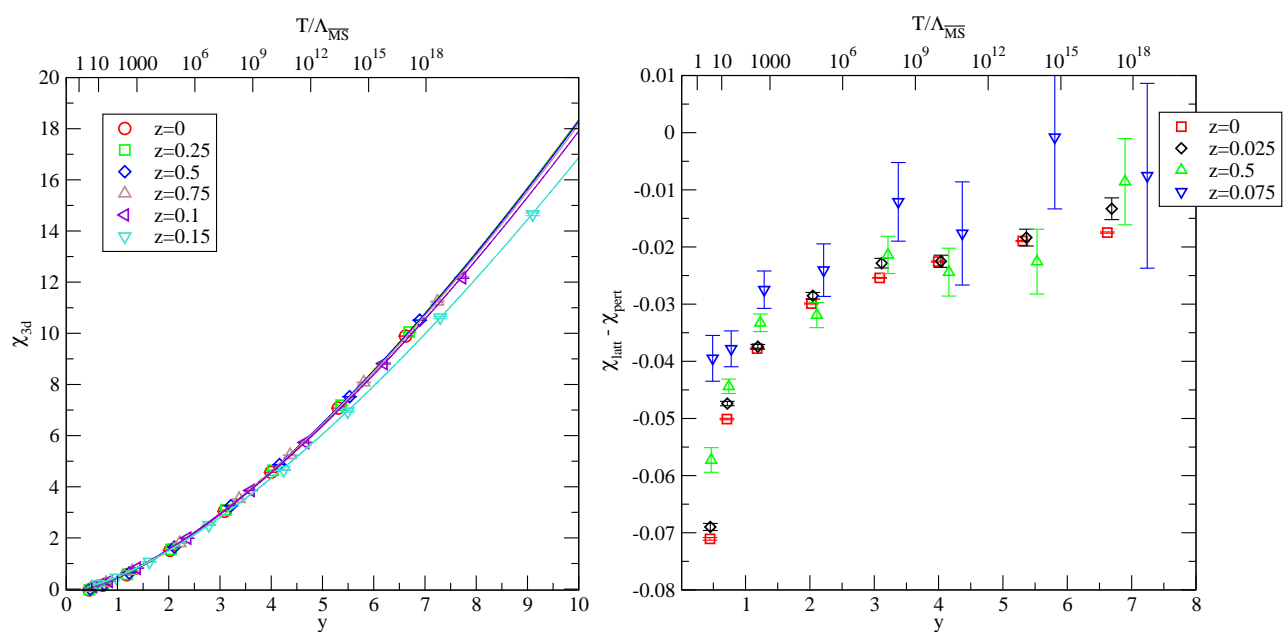

Figure 4: Left: The quark number susceptibility in EQCD, with different values of chemical potential in dimensionless units. The solid lines are the perturbative result. Right: The difference between the perturbative and lattice results. (The $T$-scale on the top of the figures corresponds to $z=0$ case.)

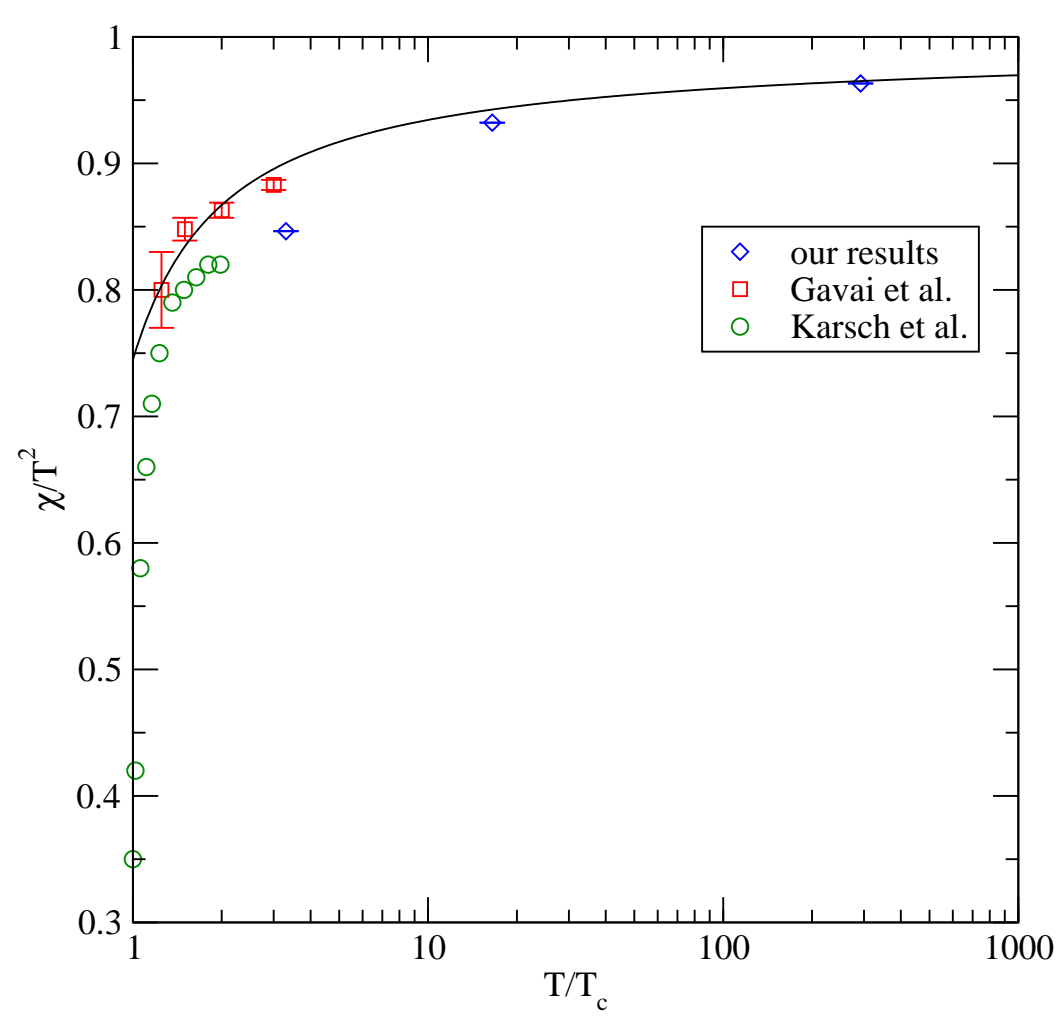

Figure 5: The susceptibility in $4 \mathrm{~d}$ units at $\mu=0$. The results agree with $4 \mathrm{~d}$ lattice simulation results. 
After the matching to $4 \mathrm{~d}$ QCD we obtain the susceptibility in physical units. Our results significantly deviate downwards from the perturbation theory, as can be seen from Fig. 5, bringing the results closer to the recent simulations by Karsch et al. [4]. However, one should bear in mind that our results suffer from a matching ambiguity related to the unknown $O\left(g^{6}\right)$ coefficient in perturbation theory, see Fig. 1, and the results need to be matched to a known point (4d lattice simulation) at some low temperature. Nevertheless, we can say that the deviation from perturbative result is still rather large at $10 T_{c}$. See Fig.5.

\section{Acknowledgments}

This work has been partly supported by the Magnus Ehrnrooth Foundation, a Marie Curie Felloswhip for Early Stage Researchers Training, and the Academy of Finland, contract number 104382. Simulations have been carried out at the Finnish IT Center for Science (CSC).

\section{References}

[1] M. Asakawa, U. W. Heinz and B. Muller, Fluctuation probes of quark deconfinement, Phys. Rev. Lett. 85 (2000) 2072 [arXiv:hep-ph/0003169].

[2] A. Vuorinen, Quark number susceptibilities of hot QCD up to $g * * 6 \ln (g)$, Phys. Rev. D 67 (2003) 074032 [hep-ph/0212283].

[3] R. V. Gavai, S. Gupta and P. Majumdar, Susceptibilities and screening masses in two flavor QCD, Phys. Rev. D 65 (2002) 054506 [hep-lat/0110032].

[4] F. Karsch, S. Ejiri and K. Redlich, Hadronic fluctuations in the QGP, Nucl. Physifcon. A 774 (2006) 619 [hep-ph/0510126].

[5] K. Kajantie, M. Laine, K. Rummukainen and M. E. Shaposhnikov, 3d $S U(N)+$ adjoint Higgs theory and finite-temperature QCD, Nucl. Phys. B 503 (1997) 357 [hep-ph/9704416].

[6] K. Kajantie, M. Laine, K. Rummukainen and Y. Schroder, How to resum long-distance contributions to the QCD pressure?, Phys. Rev. Lett. 86 (2001) 10 [arXiv:hep-ph/0007109].

[7] A. Hietanen and K. Rummukainen, Quark number susceptibility at high temperature, PoS LAT2006 (2006) 137 [arXiv:hep-lat/0610111].

[8] A. Hart, M. Laine and O. Philipsen, Static correlation lengths in QCD at high temperatures and finite densities, Nucl. Phys. B 586 (2000) 443 [hep-ph/0004060].

[9] A. Vuorinen, The pressure of QCD at finite temperatures and chemical potentials, Phys. Rev. D 68 (2003) 054017 [hep-ph/0305183].

[10] A. Hietanen, K. Kajantie, M. Laine, K. Rummukainen and Y. Schroder, Plaquette expectation value and gluon condensate in three dimensions, JHEP 0501 (2005) 013 [hep-lat/0412008];

A. Hietanen and A. Kurkela, Plaquette expectation value and lattice free energy of three-dimensional $S U(N(c)$ gauge theory, hep-lat/0609015.

[11] C. Torrero, M. Laine, Y. Schroder, F. Di Renzo and V. Miccio, Renormalization of infrared contributions to the QCD pressure, PoS LAT2006 (2006) 038 [arXiv:hep-lat/0609048];

C. Torrero, F. Di Renzo, M. Laine, V. Miccio and Y. Schroeder Four-loop NSPT result for a 3d condensate-contribution to hot QCD pressure, PoS LAT2007 (2007) 231. 